\title{
Local Knowledge and Single IRBs for Multisite Studies: Challenges and Solutions
}

\author{
Robert Klitzman, Ekaterina Pivovarova, Alexandra Murray, \\ Paul S. Appelbaum, Deborah F. Stiles, and Charles W. Lidz
}

ABSTRACT New federal policies require single IRB review for multisite studies, but many questions remain about how these IRBs will use local knowledge. The findings from our study, the first to examine how single IRBs perceive needs for local knowledge, reveal several challenges. Study respondents identified four potentially relevant types of local knowledge: about culture and linguistics, about geography and socioeconomics, about the researchers, and about the institutions. Such knowledge can potentially be obtained through local sites, but single IRBs may be unaware of potentially relevant local information, and lack of informal relationships may impede single IRBs' reviews and interactions with researchers. While a recent, commonly used, standardized single-IRB form asks three basic questions about local information, our findings suggest potential needs for additional information and, thus, have important implications for practice, policy, and research. KEYWORDS multisite studies, single IRBs, local IRBs, Common Rule, research ethics, human subjects research Klitzman, R., et al., "Local Knowledge and Single IRBs for Multisite Studies: Challenges and Solutions," Ethics \& Human Research 41, no. 1 (2019): 22-31. DOI: 10.1002/eahr.500003

Wo U.S. federal policies adopted recently mandate the use of a single institutional review board (IRB) for most federally funded multisite studies: one issued by the National Institutes of Health (NIH) and the other by the Department of Health and Human Services (DHHS) as part of its changes to the regulations governing human subjects research (the Common Rule). ${ }^{1}$ Proponents of the single-IRB approach contend that it will reduce delays and investigator burden in the review process, save resources, and enhance consistency in reviews while adequately accounting for local knowledge issues. ${ }^{2}$

However, concerns persist that important input from study sites may be lost by eliminating "local IRB" review from those sites. In comments submitted in response to DHHS's proposal in $2011^{3}$ for the singleIRB mandate and its revised proposal in $2015,{ }^{4}$ some respondents noted that local IRBs can uniquely provide cultural context, address community concerns and needs, enhance communication with investigators, and manage legal risks. ${ }^{5}$ For example, George Annas from Boston University argued that

[n]o part of the federal government, let alone the agency whose purpose is the protection of human subjects, should even attempt to require institutions to surrender their desire and ability to protect their research subjects as a condition of eligibility to participate in a multicenter trial ... . Mandated outsourcing of IRB review cannot further protect research subjects. It undermines the entire rationale for the creation of IRBs themselves: the responsibility to protect the local community and its members who may become research subjects. ${ }^{6}$

Another respondent, Matthew Hodgson, the assistant vice president of research compliance and regulatory affairs at the Children's Hospital of Philadelphia, pointed out that "as a pediatric institution, we encounter proposals for a central IRB where the proposed [r] eviewing IRB has little or no discernible experience with pediatric research."7

The National Center for Advancing Translational Sciences has developed the Streamlined, Multisite, Ac- 
celerated Resources for Trials IRB Platform (SMART IRB) as a systematic approach to single-IRB review, ${ }^{8}$ but the local-issues form suggested by SMART IRB includes only three questions for collecting local information: "Are there any state laws that the single IRB will need to consider when reviewing the study?", "Are there any community or cultural differences for the local population of subjects that require consideration?", and "Is 18 the age of majority in the state where each research site is located?"9 To date, the few studies that have examined central IRBs (CIRBs), a type of single IRB the National Cancer Institute helped implement, have focused exclusively on issues of efficiency (such as costs and the time to the start of the study). No published research directly examined quality of review, including knowledge of and attention to local issues. A recent review concluded that more data were needed and that the United States should look to examples of CIRBs in various European countries. ${ }^{10}$ However, because these countries generally have considerably smaller geographic areas, populations, and research programs than the United States does, CIRBs in those settings may have fewer difficulties obtaining important local knowledge.

The purpose of our study was to understand the experiences and perspectives of key IRB personnel regarding issues about local knowledge in the IRB review process to learn what kind of knowledge, if any, single IRBs will need in reviewing protocols for multisite studies. Although we recognize that the new mandated singleIRB approach differs in some ways from the use of the CIRBs that were in place when the single-IRB polices were issued, the intent of existing CIRBs and the new single-IRB model is to minimize the number of IRBs reviewing the same protocol, and the terms "central IRB" and "single IRB" are often used interchangeably. Thus, in this article we use the term "single IRB" when describing our study's methods and findings.

\section{STUDY METHODS}

Te conducted in-depth interviews with indi7 viduals involved with single IRBs for multisite studies. To identify eligible single IRBs from which to recruit participants, we relied on lists from the following six resources: Association for the Accreditation of Human Research Protections Programs; ${ }^{11}$ the Office for Human Research Protection (OHRP) Program's registration portal for IRBs; ${ }^{12}$ Citizens for Responsible Care and Research, which compiles a list of commercial IRBs; ${ }^{13}$ the Public Responsibility in Medicine and

\begin{tabular}{|c|c|c|}
\hline \multicolumn{3}{|c|}{$\begin{array}{l}\text { Demographic Background } \\
\qquad N=103\end{array}$} \\
\hline Age (in years) ${ }^{a}$ & $n$ & $\%$ \\
\hline $20-39$ & 20 & 19.4 \\
\hline $40-49$ & 21 & 20.4 \\
\hline $50-59$ & 25 & 24.3 \\
\hline $60+$ & 29 & 28.2 \\
\hline \multicolumn{3}{|l|}{ Gender } \\
\hline female & 65 & 63.1 \\
\hline male & 38 & 36.9 \\
\hline \multicolumn{3}{|l|}{$\operatorname{Race}^{b}$} \\
\hline black & 5 & 4.9 \\
\hline white & 91 & 88.3 \\
\hline Latino(a) & 3 & 2.9 \\
\hline other & 1 & 1.0 \\
\hline \multicolumn{3}{|l|}{ Role } \\
\hline chair & 20 & 19.4 \\
\hline director & 27 & 26.2 \\
\hline member & 30 & 29.1 \\
\hline staff & 26 & 25.2 \\
\hline \multicolumn{3}{|c|}{ Educational background ${ }^{c}$} \\
\hline bachelor's or less & 31 & 30.1 \\
\hline MA or MS & 28 & 27.2 \\
\hline $\mathrm{JD}$ & 4 & 3.9 \\
\hline $\mathrm{MD}$ or $\mathrm{DO}$ & 24 & 23.3 \\
\hline PhD or PsyD & 11 & 10.7 \\
\hline PharmD & 4 & 3.9 \\
\hline \multicolumn{3}{|c|}{ IRB Experience (in years) ${ }^{d}$} \\
\hline$<1$ & 3 & 2.9 \\
\hline $1-4$ & 22 & 21.4 \\
\hline $5-9$ & 23 & 22.3 \\
\hline $10-19$ & 29 & 28.1 \\
\hline $20+$ & 16 & 15.5 \\
\hline \multicolumn{3}{|c|}{$\begin{array}{l}\text { Note: Missing participant data for } a=8 \text {, for } b=3 \text {, for } c=1 \text {, an } \\
\text { for } d=10 \text {. }\end{array}$} \\
\hline
\end{tabular}


Research (PRIM\&R) website; ${ }^{14}$ the relevant published literature; and personal communications with local and single IRBs. We excluded IRBs that did not currently serve as single IRBs, had inactive status, were not registered with OHRP, or were based outside of the United States. We included independent (also commonly referred to as "for-profit" or "commercial") IRBs and not-for-profit IRBs, which included IRBs used in federal agencies and academic institutions, since both serve as single IRBs. In total, we contacted 49 IRBs determined to be eligible for participation in this study, of which 30 were independent and 19 were not-for-profit. Ultimately, we enrolled seven for-profit IRBs (23.3\% of all the for-profit IRBs contacted) and 13 not-for-profit IRBs ( $68.4 \%$ of all the not-for-profit IRBs contacted). None of the four largest for-profit IRBs agreed to participate. At three of these four companies, the leadership of the IRB initially wrote letters in support of the study and agreed to participate, but changes in the ownership and leadership of the companies ultimately led to these organizations' refusals to allow their IRBs to join the study. Nonetheless, $26.9 \%$ of the other forprofit IRBs (7/26) participated.

Once an IRB was enrolled in the study, we sought to interview all personnel with knowledge and experience about single-IRB processes, including directors or business managers, chairs, reviewers, and staff members (such as IRB analysts, quality assurance personnel, and liaisons). To minimize the burden on the IRB, we used the recruitment format that the site preferred. For instance, some sites preferred that we send a recruitment packet, including a letter describing the study, procedures for the interview, and a fact sheet, which they distributed to their staff and IRB members. Others opted to provide us with email contacts or phone numbers for individuals who might be interested in participating.

The interview protocol was informed by prior research with IRB personnel and members ${ }^{15}$ and by pilot interviews with the chair and staff members of a local IRB. The interview broadly examined (1) sIRB processes; (2) changes to IRB policies (NIH and Common Rule proposed and subsequently adopted regulations); (3) conflict of interest; (4) relationships with local institutions, researchers, and funders; (5) differences in single-IRB, CIRB, and local-IRB reviews; and (6) organizational issues related to single-IRB operations. Four versions of the semistructured interviews were used to best reflect experiences of the interviewees: administrators, chairs, members, and staff members. Additionally, all participants were asked to provide basic demographic information.

The interviews were oral, all were transcribed, and identifying information (such as site, location, and names) was redacted. A detailed codebook was developed based in part on a previous NIH-funded study of local IRBs ${ }^{16}$ and was modified using findings from pilot and initial interviews for the study. The development of the codebook was an iterative and collaborative process involving all investigators and three coders.

Given the variable recruitment process, the number of individuals contacted for interviews could not be ascertained. In total, we interviewed 103 participants from 20 different IRBs. Respondents were predominantly female $(\mathrm{n}=65 ; 63.1 \%)$; identified as white $(\mathrm{n}=$ 91; 88.3\%) (see the table for demographic information), and held the following IRB roles: chair $(\mathrm{n}=20 ; 19.2 \%)$, director or business manager $(\mathrm{n}=27 ; 26.2 \%)$, member $(\mathrm{n}=30 ; 29.1 \%)$, or staff member $(\mathrm{n}=26 ; 25.2 \%)$. Most participants were interviewed by phone $(n=92 ; 89.3 \%)$. All interviews were conducted by two of the authors, a licensed clinical psychologist and a board-certified psychiatrist. The participants were offered a $\$ 20$ gift card or cash (if the interview was conducted in person) for their time; many, however, declined to accept payment.

\section{IMPORTANCE OF LOCAL KNOWLEDGE}

$\mathrm{M}$ any respondents felt that the importance of local knowledge was overstated and that potential gaps in local knowledge were not very important. Others suggested that although the IRB at each study site may know local issues better than the single IRB of record, the advantages of a single-IRB approach outweigh the potential disadvantages of insufficient local input and that such input can be readily incorporated into the single-IRB review process. As one respondent noted, "I don't really feel one way or the other on local context, but I just know that's what people [cite as] the reason not to embrace the CIRB concept .... We don't write one set of stipulations for Hospital A and another one for Hospital B .... I don't even think we think like that. So I think the local context thing might not . . . 
hold as much water as people would like it to" (Chair 3, Academic IRB 11).

Several respondents believed that only minimal, if any, differences in legal and cultural norms exist across all 50 states. According to one respondent, "When you decide to deal with international [studies] . . . differences in culture might play a role. But as far as CIRBs in the U.S., I think that there shouldn't be a great deal of difference in culture from Massachusetts to California to Indiana to Iowa" (Member 1, Independent IRB 6). And some respondents suggested that local differences may involve only relatively small matters, often just different approaches to the content of consent forms. One respondent explained that hospitals may have different consent language because their legal counsel was involved, but that such differences are "usually inconsequential" (Chair 1, Independent IRB 4). Another noted that many local IRBs have relied on independent CIRBs for certain protocol reviews for many years.

Some respondents also pointed out that local differences may be less important now than in the past. Said one respondent, "Over the years, as everything has matured, in some areas we're realizing that either there isn't as much variation or there shouldn't be as much variation" (Director 1, Academic IRB 2). As to concerns about differences in state laws, respondents noted that differences can often be easily handled. For instance, a single IRB providing oversight for a study site in California could require that the state's participant bill of rights be attached to the consent form, and protocols could be easily amended to address differences in state laws regarding the age of majority. With regard to differences in state laws that have restrictions on certain types of research, such as fetal research or studies using biospecimens, one responded said that the "local site can decide whether or not it's appropriate to participate... . I think that those [matters] are easily handled" (Member 1, Academic IRB 12). Another respondent felt that resistance to single-IRB review may reflect concerns about the local IRBs' "turf" and loss of control of research at their own institutions. Concerns are often "oververbalized as a means of protecting turf. I think [local variations were] probably truer when the regulations were first written, but now ... everybody has the same Starbucks ..." (Chair 1, Academic IRB 13).
Although many respondents thought that concerns that single IRBs will not adequately address issues related to local knowledge were often overstated, some provided examples of local issues that might be relevant for the single IRB to know about. These included cultural and linguistic characteristic of potential participants, geographic and socioeconomic issues, knowledge about particular researchers, and information about institutional differences.

Cultural and linguistic issues. The range of cultural practices in a community and languages spoken can be a vital type of local knowledge about which IRBs need to be aware. For many potential participants, English may be a second language or barely spoken at all. The

\section{Relevant local information includes} cultural and linguistic characteristics of potential participants, geographic and socioeconomic issues, knowledge about researchers, and information about study-site variation.

variety of primary languages can be wide (including, for example, Mandarin, Somali, Haitian Creole, Vietnamese), and there may be a need to translate consent forms, questionnaires, and other study materials into relevant languages. One respondent said one local area from which they recruited participants had a large population that spoke only Navajo. Since translating consent forms and study materials and finding individuals to translate during recruitment and consent sessions can involve considerable costs, researchers might exclude non-English-speaking individuals for these studies. Some respondents questioned whether the single IRB could manage this issue successfully. As one respondent pointed out, "People from the [local] IRBs can really bring information and perspectives that the central group can't possibly have, for example, how to increase diversity among the participants in the study. Frankly, I think that could only be handled locally, because only 
local people know ... the landscape with respect to the potential participants" (Chair 1, Independent IRB 6).

Knowledge of a specific culture can also be important in understanding the behavior of research participants in ways that may be relevant to the study. One independent IRB director described the problem with a medication study that required eating a specific high-fat diet to facilitate drug absorption, so fish was not on the menu. The director noted that adherence to the protocol was especially problematic in their area because a large percentage of participants were Catholics who refused to eat meat on Friday.

Geographic and socioeconomic issues. Local IRBs may be aware of relevant aspects of a region that may affect the safety or burden faced by study participants. For example, whereas in cities like New York, participants who are outpatients can use the subway to make extra clinic visits, participants in a rural area may be burdened by an hour-and-a-half drive to a medical center to make an extra visit for a study (Chair 1, Independent IRB 4). Other socioeconomic factors include rates of poverty in certain areas, which can be relevant because of the risk of undue influence from financial enrollment incentives and the consequent potential for inappropriately burdening a vulnerable population. As one respondent explained, "Somebody doing research in the Bay Area [may] have a site in Mississippi. And, in the Bay Area, they're gonna give each person $\$ 100$. In the Bay Area, $\$ 100$ is not a lot of money .... Food costs are very high. Then we get the person from Mississippi who's like, 'Ehhh, that's kind of coercive here,' because that's a lot of money [in Mississippi]" (Chair 1, Independent IRB 1).

Researchers. Some respondents felt that the local IRB's "personally knowing" the principal investigator (PI) of a study could be advantageous for several reasons. First, personal interactions can facilitate and streamline protocol review, whereas formal interactions (such as via memos), which might occur more often with single IRBs, may be less effective (Staff Member 7, Academic IRB 8). And certain PIs can be overextended in ways that may raise concerns. Awareness of how many other studies a researcher or clinic is conducting may allow a local IRB to assess whether a research group at its institution can realistically take on a new study. According to one respondent, when a PI says she or he has "20 studies that they're doing right now .... [t]hat's a big red flag for me-How do you think you can manage this study? Tell me where you are in the process of your other studies. Are they winding down?" (Director 1, Independent IRB 7).

An additional way in which local knowledge about researchers is important concerns particular researchers' histories of noncompliance, including a range of "misbehaviors" that may not constitute serious noncompliance but are nonetheless of concern, ${ }^{17}$ such as "cutting corners" in following human protection regulations. ${ }^{18}$ "I have a very good grip on our system and our investigators," said one respondent. "I know them ... and I know the 'turkey' list. I know the well-meaning but clueless and the people who are always trying to shortcut. That's lost-that does not come through at all" (Chair 1, Academic IRB 1). And written information provided to the single IRB in the protocol or other information, such as documentation of training in the responsible conduct of research, might not fully or sufficiently convey these concerns. One respondent gave an example about an investigator with "serious continuing noncompliance":

The hospital knows the investigator. So [if] you're looking at it on paper, it looks fine. Everything looks good, and he's saying he's going to do X, Y, and Z. Then you call, or a medical center might call us and say, "Do you have the so-and-so study?"

"Yup."

"Watch out for this guy!" (Chair 2, Independent IRB 4)

Another respondent involved with a local IRB pointed out that single IRBs may have to rely only on documentation of responsible conduct of research training, which may be insufficient. "I would know whether or not they knew what they were doing" (Director 1, Independent IRB 7). But without that local contact, as a single IRB, this repondent went on to say, "[w]e have to base our opinions [on] basically how well they fill out the information. I have to take their word sometimes for what they're telling me." Finally, local knowledge of PIs can affect how closely an IRB reviews or monitors a PI's protocols over time. As one respondent pointed out, "There have been times where we've had history with a specific investigator. A study has been submitted by that investigator, and based on our history, we might decide to review their research more 
frequently ... or provide closer monitoring, request external monitoring" (Director 1, Academic IRB 12).

However, some respondents saw a virtue in single IRBs' having less direct contact with investigators. "The lack of 'personal relationships' ... is also helpful in some ways," a respondent said, "because you don't have people trying to use their influence in some way" (Staff Member 7, Academic IRB 8). In the words of another respondent, "Being able to be objective is easier for a CIRB. To be objective and not worry about hurting someone's feelings or something like that" (Chair 1, Independent IRB 1).

Institutions. Knowledge of different institutional structures, policies, and procedures (such as radiation safety or biosafety and biospecimen handling) can also be relevant for IRBs. Institutional factors may affect, for instance, the availability of particular medications in a pharmacy. One respondent said, "[B] eing a pharmacist, I think about this all the time: the local drug supply, the local support from the pharmacy, whether ... they thought about what drugs were available at all the different sites" (Chair 1, Academic IRB 13).

Respondents also pointed out that differences in institutional policies, cultures, and standards of care could have relevance for disclosure of information in consent forms. Researchers at a hospital affiliated with a religious institution "may not be able to include specific birth control references" in a consent form (Director 1, Academic IRB 12). And some institutions may have different template language for consent forms about how research biospecimens are handled or have specific "HIPAA language, things like that" (Chair 1, Academic IRB 8). There may also be particular differences in legal language regarding risk and liability. One IRB chair said, "[U]nfortunately, we have different paragraphs for different IRBs because different hospitals define risk differently, so they have different statements" (Chair 2, Independent IRB 4). Yet one respondent said it would depend on the specific type of study as to whether such differences are relevant: "A lot of the research that would go to a CIRB might be okay if this was another diabetes drug, or another heart disease drug, or another stent ... and local context issues have very little influence on the conduct" (Director 1, Academic IRB 12).

\section{MECHANISIMS TO OBTAIN LOCAL KNOWLEDGE}

T $\mathrm{f}$ a single IRB of record should have knowledge of these and other local issues, how can such information be obtained? As noted above, the SMART IRB, one of the leading efforts to address the single-IRB mandate, has proposed a form for IRBs at local study sites to complete with only three questions that address local issues. A variety of other approaches have been developed. Local information forms developed by single IRBs, for instance, can include information on race or ethnicity of local populations, relevant local laws, and even information about the PI. One respondent said that their protocol review application asks researchers, "Are there local issues? What are the demographics of the community versus who you are targeting?" (Chair 1, Independent IRB 1). Another explained that in their

local site investigator applications, the one place where we'll really hold the sites up is if we don't have good documentation that this person has done clinical trials in a recent timeframe or at all. We ask for mentoring relationships ... [and] we also have kind of a fallback as well in that we have a research service at each site, and the associate chief of staff for research has to sign off on that investigator. (Chair 1, Academic IRB 13)

Individuals can also be appointed to serve as liaisons between single IRBs and local sites, something we observed at many federal and academic single IRBs, which can help in obtaining relevant local information. A single IRB may have other formal links with its sites as well. As one independent IRB director reported, "We also have research compliance officers at every research location that, not before the study, but after the study is ongoing, do audits either for cause, or no matter what" (Director 1, Independent IRB 7)-in other words, routinely. Respondents noted that they can obtain more information about local issues by asking a lot of questions (Chair 1, Academic IRB 7) and even conducting Internet searches to enhance their understanding of local contexts and populations, as well as to get information about the PI's credentials (Member 4, Independent IRB 1). For instance, one respondent remarked,

The Internet has helped .... We can go online and see where they are at .... There's so much information about where all these PIs are . . I can look at their building through Google Doc, maps. So it's no longer: "Oh wow, we don't know the local conditions ..." "Oh, I've never 
been to Nebraska! How is it there?" ... . That's not an issue.... Before, it was more like a fax report. ... We would get a fax. . . . But now. . . I could see what you're doing practically. . . in a really rural and really poor place. (Staff Member 3, Independent IRB 1)

Nevertheless, there may be challenges obtaining local input because single IRBs, local IRBs, and PIs may differ in quality, attitudes, and expertise and in their willingness to collect and share local knowledge. In particular, problems may occur when attempting to collect information about very large studies with many sites. Single IRBs should have effective and efficient mechanisms for collecting necessary information. But some local sites may routinely provide little if any information, comments, or feedback. According to one respondent, "Some institutions just review it and sign off on things, and some look at things a lot more closely" (Director 1, Academic IRB 9). Another said that "[a] few facilities are sort of consistent contributors [compared to] other sites we rarely hear from" (Chair 2, Nonprofit IRB 13, Government). Single IRBs may also have trouble obtaining relevant information about local researchers. Some respondents said they relied on the fact that PIs are generally vetted by the study sponsors. Yet the qualities the sponsors look for in PIs may not be the same as those that IRBs ideally should examine (for example, training in human subjects protections or record of adherence to IRB requirements). Finally, local sites may not at first provide important information, requiring proactive inquiry by the single IRB. One respondent explained, "We look at things like is there a large Spanish-speaking population in that area? ... Even if it's not brought up by the local site, we'll ask that question" (Director 1, Academic IRB 13). Whether a local population is being overstudied may be particularly difficult for single IRBs to ascertain.

\section{RECOMIMENDATIONS}

${ }^{0}$ our knowledge, our study is the first to present 1 findings about how single IRBs acquire and use local knowledge in their review of protocols for multisite studies. Although some study respondents felt that local knowledge was less important than in the past (due to increased cultural homogenization in the United States and information available on the Internet) or could easily be dealt with through existing procedures, others provided examples of how complex the issues can be. Four broad types of local information were cited as relevant to single-IRB review: cultural and linguistic characteristics of potential participants, geographic and socioeconomic issues, knowledge about particular researchers, and information about differences across the study sites in a multisite study.

In theory, these types of information could be obtained through questionnaires, liaisons, and additional queries, but as we have seen, these efforts are not always straightforward. The quality of local knowledge provided by local IRBs and PIs may vary, based on how well these parties recognize what information might be necessary to provide to the single IRB. Importantly, informal interactions with local PIs, which could answer these questions, may be less likely to occur with the use of single IRBs. These issues will be increasingly important to address since the new single IRBs will be evaluating larger numbers and more diverse types of protocols, patient populations, communities, researchers, and institutions.

The findings from our study have important implications for research and policy about the single-IRB approach for multisite studies. Although the SMART IRB form asks about three basic issues, our data suggest that, in the review of many studies, additional information may be important. Based on our findings, additional questions should be considered for inclusion in documentation that local IRBs provide the single IRB of record:

- Linguistic and cultural issues: For what percent of potential participants is English not a primary language? Has the protocol appropriately considered the possibility that English will not be the primary language for all potential participants and made any necessary provisions? Are there dietary or other religious or cultural restrictions for the population that are relevant to the study?

- Geographic and socioeconomic issues: Are there geographical issues (such as distances to study sites, clinics, or emergency rooms for some participants) that should be considered? What is the cost of living in the area relative to participant payment? Will limited access to specialists in the area or to treatment for conditions being studied create undue incentives for participation or burdens?

- Researchers: Is there a history of noncompliance or difficulties in obtaining information from this re- 
searcher or the research team? Has this researcher ever conducted other multisite research studies, and if so, have there been any problems in complying with regulations or IRB requirements? How many other studies is the PI or the research team managing at this time? Have there been concerns with this researcher that have not been formally documented?

- Institution-specific issues: Are there pharmacyspecific issues (for example, access to medication or pharmacy hours) that will need to be addressed prior to starting this study? Is this a hospital that serves a population with a dominant religious orientation, and if so, does that affect discussions of birth control, pregnancy, and so forth? Are there procedures that are site-specific that may affect the study (such as the process for obtaining consent during emergency procedures)?

Our findings also highlight the need for single IRBs to triangulate information and not rely only on answers on forms to make optimal decisions about whether to monitor certain PIs or protocols more carefully than others and, if so, how. The findings also suggest possible benefits of having single IRBs be as proactive as possible, scanning for potential problems, while recognizing that challenges may arise due to local circumstances that cannot always be fully foreseen.

Increasingly, academic centers constitute large, sprawling health care systems, consisting of many diverse hospitals and clinics throughout a geographic area that may vary considerably in patient populations. A PI at an academic medical center may be recruiting from several types of hospitals, clinics, or provider offices. Hence, the single IRB may need to request data not only about the researcher's institution but also for each specific recruitment site. Though critics may wonder whether sites will want to assemble such data, many local IRBs have argued that single IRBs' knowledge of such local information is critical for many (but not necessarily all) studies. Such knowledge can help to ensure that ethical and regulatory requirements for research are followed, for instance, that study participants provide appropriate and full informed consent, that they are not subject to coercion or undue influence, and that risks due to potential violations of research integrity are minimized. State laws, for example, can vary concerning age of consent and handling of biological material (genetic testing, handling of biospecimens, and han- dling of and research on fetal tissues), and the variations among them may pose important challenges.

Whatever procedures are developed should be iterative, with tracking of the frequency and type of additional information single IRBs request, need, and use. Research institutions could take a cue from "learning health systems," in which ongoing practice and research are closely intertwined in academic medical centers, each advancing the other to improve systems as much as possible. ${ }^{19}$ Similarly, we should strive to foster "learning single IRBs" that agree to share data and be studied to find ways to address challenges that emerge in this new, relatively untested system. Our own experience and, anecdotally, that of other researchers suggest that many large for-profit IRBs and single IRBs may decline to be studied, especially through direct observation of their meetings or minutes. Since single IRBs are mandated for most federally funded studies, policy makers ideally should require that all single IRBs provide data on practices and functioning to allow and facilitate improvement and refinement of policies for human subjects protection. Based on such data, the NIH, OHRP, the Secretary's Advisory Committee on Human Research Protections, PRIM\&R, and other relevant organizations should consider developing guidance to establish standards and "best practices" for obtaining and incorporating local information.

In addition, our findings suggest that, partly because single IRBs need to obtain sufficient input from local sites and assembling this may require considerable effort, single-IRB review may not reduce costs as much as many proponents have imagined. In many instances, the local institution retains the responsibility for monitoring and assuring compliance (for example, concerning disclosure of conflicts of interest, compliance with training requirements, pharmacy logistics, and assessment of research participants' decisional capacity). However, there are several grey areas with regard to the distribution of responsibilities between local IRBs and compliance officers. Single IRBs may also need to decide whether and how to take into account a PI's past compliance difficulties.

This study has several possible limitations. Although our sample reflected a broad range of single IRBs, it was not strictly representative, and the unwillingness of the largest for-profit single IRBs to participate limited our 
ability to reflect the views of their members and staff. We did not interview IRB personnel who were involved only with local IRBs; however, many of the respondents had worked on both local IRBs and single IRBs and were able to reflect on the two relevant sets of roles. Moreover, our study was informed by our extensive prior work on local IRBs. ${ }^{20}$ This study was designed to elicit the range of themes that emerged concerning the single-IRB approach, and the data revealed that these for-profit and not-for-profit single IRBs raised many similar issues concerning the need for single IRBs to obtain various kinds of local knowledge relevant to the research sites in a multisite study. However, our study was not designed to test differences between types of IRBs (for example, for-profit versus not-for-profit, large versus small, or urban versus nonurban). Future research could attempt to assess such differences, although it will need to take into account the hesitancy of for-profit IRBs to participate in research.

Robert Klitzman, MD, is a professor of psychiatry and the director of the Master of Science in Bioethics Program at Columbia University; Ekaterina Pivovarova, $\mathrm{PhD}$, is an assistant professor of psychiatry at the University of Massachusetts Medical School in Worcester; Alexandra Murray, BA, MPA, is a research coordinator II at the University of Massachusetts Medical School in Worcester; Paul S. Appelbaum, MD, is the Dollard professor of psychiatry, medicine, \& law and the director of the Center for Law, Ethics, and Psychiatry at Columbia University; Deborah F. Stiles, JD, is the vice president for research operations and policy and the chief operating officer in the Office of the Executive Vice President for Research at Columbia University; and Charles W. Lidz, $\mathrm{PhD}$, is a professor emeritus of psychiatry at the University of Massachusetts Medical School in Worcester.

\section{ACKNOWLEDGMENTS}

The authors would like to thank Kristina Khan Hosi, Charlene R. Sathi, and especially Patricia Contino for their assistance in preparing this manuscript.

Funding for this study was supported by the $\mathrm{Na}$ tional Institute of General Medical Studies, with grant 5R01GM113640-03 (with Charles Lidz as the principal investigator).

\section{REFERENCES}

1. National Institutes of Health, "Single IRB Policy for Multi-site Research," in Policy \& Compliance, 2017, https:// archives.nih.gov/asites/grants/08-22-2017/policy/clinicaltrials/single-irb-policy-multi-site-research.htm; Federal Register, Federal Policy for the Protection of Human Subjects (January 19, 2017), https://www.federalregister.gov/documents/2017/01/19/2017-01058/federal-policy-for-the-protection-of-human-subjects.

2. Resnik, D. B., "Centralized Institutional Review Boards: Assessing the Arguments and Evidence," Journal of Clinical Research Best Practices 11, no. 5 (2012): 1-13.

3. U.S. Department of Health and Human Services, Human Subjects Research Protections: Enhancing Protections for Research Subjects and Reducing Burden, Delay, and Ambiguity for Investigators Proposed Rules, Advanced Notice of Proposed Rulemaking (2011) Federal Register 76 (2011), 4451231, http://www.gpo.gov/fdsys/pkg/FR-2011-07-26/html/2011 -18792.htm.

4. U.S. Department of Homeland Security et al., Federal Policy for the Protection of Human Subjects; Proposed Rules, Notice of Proposed Rule Making, Federal Register 80 (2015), 53931-54061, //www.gpo.gov/fdsys/pkg/FR-2015-09-08/pdf/ 2015-21756.pdf.

5. Regulations.gov, Comments for Docket: HHS-OPHS -2011-0005. Human Subjects Research Protections: Enhancing Protections for Research Subjects and Reducing Burden, Delay, and Ambiguity for Investigators, 2011, https://www. regulations.gov/docketBrowser? $\mathrm{rpp}=25 \& \mathrm{so}=\mathrm{DESC} \& \mathrm{sb}=\mathrm{c}$ ommentDueDate \&po $=0 \& s=$ central $\& d c t=P S \& D=H H S-O$ PHS-2011-0005; Regulations.gov, Comments for Docket: HHS-OPHS-2015-0008, Federal Policy for the Protection of Human Subjects, 2016.

6. Boston University, School of Public Health, letter to Jerry Menikoff, December 9, 2015, located at docket ID number HHS-OPHS-2015-008: Notice of Proposed Rulemaking for Human Subjects Protection (NPRM).

7. The Children's Hospital of Philadelphia, "Notice of Proposed Rule Making for Revisions to the Common Rule," Docket ID number HHS-OPHS-2015-0008, January 6, 2016.

8. SMART IRB, National IRB Reliance Initiative, https:// smartirb.org/.

9. SMART IRB, "Potential Relying Site SMART IRB Point of Contact Survey," 2018, https://smartirb.org/sites/default/ files/20170510-Relying-Site-Survey-POCs.pdf.

10. Check, D. K., et al., "Use of Central Institutional Review Boards for Multicenter Clinical Trials in the United States: A Review of the Literature," Clinical Trials 10, no. 4 (2013): 56067.

11. The Association for the Accreditation of Human Research Protection Programs, 2018, http://www.aahrpp.org/.

12. Office for Human Research Protections, 2018, IRB Registration portal, https://www.hhs.gov/ohrp/register-irbs-andobtain-fwas/irb-registration/index.html.

13. Citizens for Responsible Care and Research, August 9, 
2016, http://www.circare.org/index.htm.

14. Public Responsibility in Medicine \& Research, 2018, https://www.primr.org/.

15. Klitzman, R. L., "How Local IRBs View Central IRBs in the U.S.," BMC Medical Ethics 12 (2011): 13; Lidz, C. W., et al., "The Participation of Community Members on Institutional Review Boards," Journal of Empirical Research in Human Research Ethics 7, no. 1 (2012): 1-8.

16. Lidz et al., "The Participation of Community Members on Institutional Review Boards"; Candilis, P. J., et al., "The Silent Majority: Who Speaks at IRB Meetings?," IRB: Ethics \& $\mathrm{Hu}$ man Research 34, no. 4 (2012): 1-12.
17. De Vries R., M. S. Anderson, and B. C. Martinson, "Normal Misbehavior: Scientists Talk about the Ethics of Research," Journal of Empirical Research on Human Research Ethics 1, no. 1 (2006): 43-50.

18. Martinson B. C., M. S. Anderson, and R. De Vries, "Scientists Behaving Badly," Nature 435 (2005): 737-38.

19. Faden R. R., T. L. Beauchamp, and N. E. Kass, "Learning Health Care Systems and Justice," Hastings Center Report 41, no. 4 (2011): 3.

20. Klitzman, "How Local IRBs View Central IRBs in the U.S."; Lidz et al., "The Participation of Community Members on Institutional Review Boards.” 